\title{
Stroke in the Time of Coronavirus Disease 2019: Experience of Two University Stroke Centers in Egypt
}

\author{
Tamer M. Roushdy, Nevine M. El Nahas, Hany M. Aref, Shady S. Georgy, Amr S. Zaki, Rady Y. Bedros, \\ Hossam M. Shokri \\ Neurology Department, Faculty of Medicine, Ain Shams University, Cairo, Egypt
}

\section{Dear Sir:}

In the beginning of year 2020, the world was hit by a pandemic of coronavirus disease 2019 (COVID-19), thus posing a significant challenge to health care systems worldwide. ${ }^{1}$ Consequently, emergency doctors tend to avoid hospital admission of patients with risk factors because of the higher mor- tality rate if this population is exposed to COVID-19 infection. ${ }^{2}$ Nevertheless, non-communicable diseases, as acute stroke, are still there in the background of this pandemic and definitely need to be efficiently managed. This implies that acute stroke management pathways ought to be revised to maintain proper stroke care while protecting patients and health care providers from contracting COVID-19. ${ }^{3}$

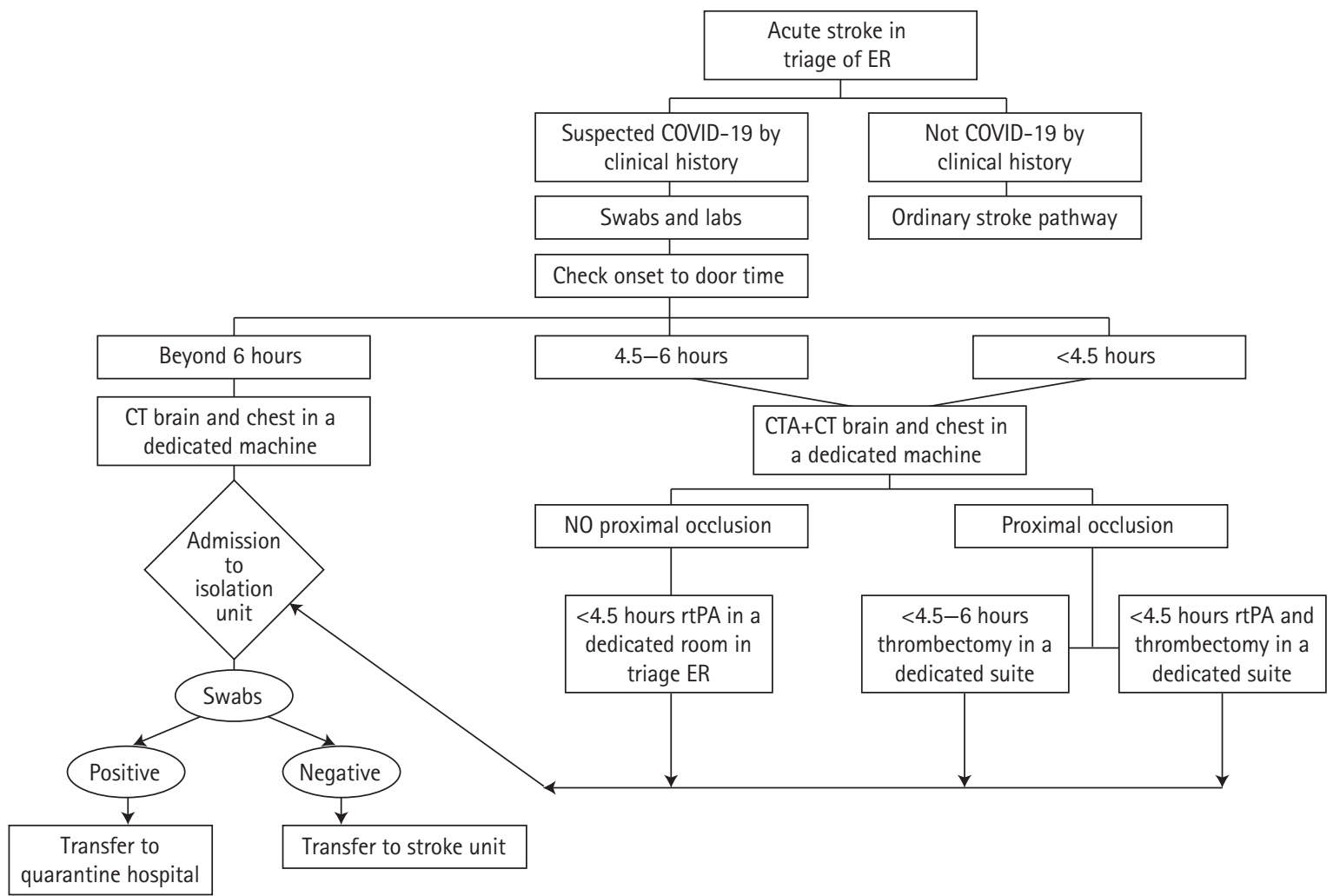

Figure 1. Modified acute stroke pathway of Ain Shams University stroke centers during the coronavirus disease 2019 (COVID-19) pandemic. ER, emergency room; CT, computed tomography; CTA, computed tomography angiography; rtPA, recombinant tissue plasminogen activator.

This is an Open Access article distributed under the terms of the Creative Commons Attribution Non-Commercial License (http://creativecommons.org/licenses/by-nc/4.0/) which permits unrestricted non-commercial use, distribution, and reproduction in any medium, provided the original work is properly cited. 
Table 1. Demographic data, treatment received, stroke subtypes and complications

\begin{tabular}{lccc}
\hline Variable & $\begin{array}{c}\text { Before COVID-19 } \\
(\mathrm{n}=151)\end{array}$ & $\begin{array}{c}\text { During COVID-19 } \\
(\mathrm{n}=93)\end{array}$ & $P$ \\
\hline Male sex & $86(57)$ & $57(61.3)$ & 0.504 \\
Age (yr) & $63 \pm 13.8$ & $63.2 \pm 12.7$ & 0.281 \\
Management & & & $0.009^{*}$ \\
Conservative treatment & $133(88.1)$ & $68(73.1)$ & \\
IV thrombolysis & $16(10.6)$ & $20(21.5)$ & \\
IV thrombolysis and & $2(1.3)$ & $5(5.4)$ & \\
$\quad$ thrombectomy & & & 0.497 \\
$\begin{array}{l}\text { Days spent in unit } \\
\text { Stroke type }\end{array}$ & $6.695 \pm 4.798$ & $6.473 \pm 4.880$ & \\
Ischemic & $138(91.4)$ & $80(86)$ & \\
Hemorrhagic & $5(3.3)$ & $7(7.5)$ & \\
Subarachnoid & $3(2.0)$ & $1(1.1)$ & \\
TIA & $5(3.3)$ & $2(2.2)$ & \\
Sinus thrombosis & $0(0)$ & $3(3.2)$ & \\
Complications & & & \\
Hemorrhagic transforma- \\
tion
\end{tabular}

Values are presented as number (\%) or mean+standard deviation. COVID-19, coronavirus disease 2019; IV, intravenous; TIA, transient ischemic attack; NA, not applicable.

*Highly statistically significant.

Table 2. Onset to door time, door to needle time, and assessment scales

\begin{tabular}{lccc} 
Variable & $\begin{array}{c}\text { Before COVID-19 } \\
(\mathrm{n}=151)\end{array}$ & $\begin{array}{c}\text { During COVID-19 } \\
(\mathrm{n}=93)\end{array}$ & $P$ \\
\hline Onset-to-door time $(\mathrm{min})$ & $600(360-900)$ & $240(128-600)$ & $<0.001^{*}$ \\
Door-to-needle time (min) & $29.6 \pm 4.9$ & $29.6 \pm 10.5$ & 0.222 \\
NIHSS baseline & $5(3-8)$ & $7(3-11)$ & $0.020^{+}$ \\
NIHSS at discharge & $4(2-6)$ & $4(2-6)$ & 0.196 \\
mRS discharge & $2(1-3)$ & $2(1-4)$ & 0.227 \\
\hline
\end{tabular}

Values are presented as median (interquartile range) or mean \pm standard deviation.

COVID-19, coronavirus disease 2019; NIHSS, National Institutes of Health Stroke Scale; mRS, modified Rankin Scale.

*Highly statistically significant; ${ }^{+}$Statistically significant.

In this study we discuss the modified pathway implemented in the two stroke centers of Ain Shams University (ASU) hospitals. We also evaluate its efficacy by comparing patient outcome over 50 days, since the declaration of the first COV-
ID-19 case in Egypt on 15th of February, with a similar immediately antedating time duration.

In this context modifications were implemented in ASU by assigning one of its three hospitals as a COVID-hospital. In the other two non-COVID hospitals, the emergency room was moved to a frontline building away from the inpatient sectors. This is where patient triage takes place with personal protective equipment provided to medical and para-medical personnel, including full sleeved gown, surgical mask, face shield and extended cuff gloves beside a N95 respirator for aerosol-generating procedures. A computed tomography (CT) machine was dedicated for suspected cases, with sterilization between sessions. Also a thrombectomy suite and an isolation unit were assigned for suspected cases. After receiving appropriate management, cases proven positive by swab are immediately transferred to the COVID hospital (Figure 1).

Statistical analysis was done using SPSS version 16 (SPSS Inc., Chicago, IL, USA). Detailed methods are available in Supplementary methods.

Outcome in COVID-19 compared to pre-COVID-19 period: despite that the total number of cases admitted in COVID period ( $n=93$ ) was less than pre-COVID ( $n=151)$, however percentage of thrombolytic therapy was significantly higher $(21.5 \%$ and $10.6 \%$ respectively, $P=0.009$ ), so was the percentage of thrombectomy (5.4\% and $1.3 \%$ respectively). As for stroke subtypes, hemorrhagic strokes and sinus thrombosis were more in the COVID period $(7.5 \%, 3.2 \%$, respectively), while in pre-COVID (3.3\%, 0\%, respectively).

Complications were notably yet non-significantly less in the COVID period (9.7\%) as compared to pre-COVID (12.6\%). Total percentage of chest infection was slightly higher in preCOVID (5.3\%) compared to (4.3\%) in COVID period. All three cases suspected to have COVID pneumonia proved negative by swab, so no patients were transferred to COVID hospital. Also death rate was more in pre-COVID (4\%) versus in COVID period (2.2\%) (Table 1).

The COVID-19 period onset-to-door time was significantly less (median 240 minutes compared to 600 minutes pre-COVID period; $P<0.001)$. However, door-to-needle time was almost equal in both time periods (Table 2).

National Institutes of Health Stroke Scale (NIHSS) baseline was significantly higher in COVID period compared to preCOVID (median 7 and 5 respectively, $P=0.02$ ). NIHSS and modified Rankin Scale (mRS) were nearly similar at discharge (Table 2). Out of 50 cases arriving beyond therapeutic window in COVID period; the commonest cause of delay was wake up stroke $30.9 \%$, while fear of catching COVID-19 or of unavailability of stroke service was $10.9 \%$. 
Three years ago, since the implementation of the acute stroke protocol in our stroke centers, the rate of revascularization therapy has increased up to about 5 folds. ${ }^{4}$ Thus, since the emergence of COVID-19 in Egypt, our main concern was to keep the acute stroke service running with the same efficiency. The results of the current study showed that some stroke patients refrained from seeking emergency treatment at hospitals for fear of infection, which explains less number of admitted stroke patients and higher NIHSS denoting severe stroke. It is possible that patients with minor stroke in the COVID period tend to decline from seeking help at hospitals. This goes with the conclusion of the European Stroke Organization in a survey among 55 countries that fear from becoming infected kept patients from seeking help. ${ }^{5}$ In spite of that the rate of thrombolytic and bridging therapy was higher possibly due to shorter transportation time and availability of caregivers due to curfew and lock down and consequently shorter onset-to-door time. While the relatively longer door-to-needle time, reaching a maximum of 60 minutes, as compared to a maximum of 40 minutes pre-COVID is attributable to more steps taken on admission including CT chest and nasopharyngeal swab.

Type of stroke did not show significant difference, yet there is a trend towards more hemorrhagic strokes and sinus thrombosis. Whether this is due to disturbance of coagulation profile attributable to undetected COVID-19, needs to be verified.

Finally, complications seem to be less during the COVID period which can be due to heightened infection control measures taken in the whole hospital. To our knowledge this is one of the first studies to set a comparative analysis of acute stroke service pre- and during the declaration of the COVID-19 pandemic.

In conclusion, acute stroke management is a dynamic process that is subject to modification according to changing and challenging situations. Implementation of a modified stroke pathway compatible with infection control measures in our stroke centers resulted in an overall outcome similar in efficiency to the pre-COVID pathway.

\section{Supplementary materials}

Supplementary materials related to this article can be found online at https://doi.org/10.5853/jos.2020.01550.

\section{References}

1. Di Gennaro F, Pizzol D, Marotta C, Antunes M, Racalbuto V, Veronese $\mathrm{N}$, et al. Coronavirus diseases (COVID-19) current status and future perspectives: a narrative review. Int J Environ Res Public Health 2020;17:E2690.

2. Baracchini $C$, Pieroni A, Viaro F, Cianci V, Cattelan AM, Tiberio $I$, et al. Acute stroke management pathway during coronavirus-19 pandemic. Neurol Sci 2020;41:1003-1005.

3. Caso $V$, Federico A. No lockdown for neurological diseases during COVID19 pandemic infection. Neurol Sci 2020;41:9991001.

4. Zakaria MF, Aref $H$, Abd EINasser A, Fahmy N, Tork MA, Fouad MM, et al. Egyptian experience in increasing utilization of reperfusion therapies in acute ischemic stroke. Int $J$ Stroke 2018;13:525-529.

5. van der Worp B, Sandset EC, Dichgans M, Caso V. Likely increase in the risk of death or disability from stroke during the COVID-19 pandemic. European Stroke Organisation. https:// eso-stroke.org/likely-increase-in-the-risk-of-death-or-disability-from-stroke-during-the-covid-19-pandemic/. 2020. Accessed April 20, 2020.

\footnotetext{
Correspondence: Hossam M. Shokri

Neurology Department, Faculty of Medicine, Ain Shams University, 38 Abbasid PO 11591, Cairo, Egypt

Tel: $+20-1283561099$

Fax: +20-224346766

E-mail: hossam.shokri@med.asu.edu.eg

Received: May 2, 2020

Revised: May 5, 2020

Accepted: May 5, 2020

This study was carried out in the both stroke units of Ain Shams University hospital and Ain Shams University specialized hospital in Abbasia Square, Cairo, Egypt.

The authors have no financial conflicts of interest.
} 


\section{Supplementary methods}

Statistical analysis was done using SPSS version 16 version Statistics (SPSS Inc., Chicago, IL, USA). The Kolmogorov-Smirnov goodness of fit test was performed to test the normality of continuous data distribution. Continuous data were presented as mean and standard deviation if normally distributed and as median and interquartile range for skewed data, whereas categorical data were presented as frequencies. Regarding bivariate analysis, Student t-test was used to compare normally distributed continuous variable with nominal independent variable. Mann-Whitney $U$ test was used to compare not normally distributed continuous variable with nominal independent variable and to correlate ordinal dependent variable versus nominal independent variable. The chi square test was used for comparison of nominal data. Fisher's exact test was used if $>20 \%$ of the cells in any cross tabulation had an expected count of $\leq 5$. $P<0.05$ was considered statistically significant. 